\title{
LIMITED CAPACITY FOR AMMONIA REMOVAL BY BRAIN IN CHRONIC LIVER FAILURE: POTENTIAL ROLE OF NITRIC OXIDE
}

\author{
Christopher Rose ${ }^{1}$ and Vicente Felipo ${ }^{1,2}$
}

Laboratory of Neurobiology, Centro de Investigacion Principe Felipe, Fundacion Valenciana de Investigaciones Biomedicas, Avda del Saler, 16, Camino de las Moreras, 46013, Valencia, Spain. To whom correspondence should be addressed at Laboratory of Neurobiology, Centro de Investigacion Principe Felipe, Fundacion Valenciana de Investigaciones Biomedicas, Avda del Saler, 16, Camino de las Moreras, 46013, Valencia, Spain. E-mail: vfelipo@ochoa.fib.es

\section{ABSTRACT}

Chronic liver failure leads to hyperammonemia and consequently increased brain ammonia concentrations, resulting in hepatic encephalopathy. When the liver fails to regulate ammonia concentrations, the brain, devoid of a urea cycle, relies solely on the amidation of glutamate to glutamine through glutamine synthetase, to efficiently clear ammonia. Surprisingly, under hyperammonemic conditions, the brain is not capable of increasing its capacity to remove ammonia, which even decreases in some regions of the brain. This non-induction of glutamine synthetase in astrocytes could result from possible limiting substrates or cofactors for the enzyme, or an indirect effect of ammonia on glutamine synthetase expression. In addition, there is evidence that nitration of the enzyme resulting from exposure to nitric oxide could also be implicated. The present review summarizes these possible factors involved in limiting the increase in capacity of glutamine synthetase in brain, in chronic liver failure.

Key words: Chronic liver failure; ammonia; glutamine synthetase; nitric oxide; hepatic encephalopathy.

\section{INTRODUCTION}

There is a substantial body of evidence to suggest that ammonia has adverse effects on the brain and is therefore believed to play a major role in the pathogenesis of the neuropsychiatric disturbances observed in hepatic encephalopathy. When liver, the major ammonia-removing organ in the body, fails and is incapable of efficiently converting ammonia to urea or glutamine, hyperammonemia develops, leading to increased levels of ammonia in the brain. Unlike liver, brain is devoid of an effective urea cycle and therefore relies entirely on glutamine synthesis for the removal of blood-borne ammonia. Glutamine synthetase (GS) is strictly an astrocytic enzyme, 
Rose, C. \& Felipo, V., 2005. Limited capacity for ammonia removal by brain in chronic liver failure: potential role of nitric oxide. Metabolic brain disease, 20(4), p.275-83.

which is responsible for the adenosine triphosphate (ATP)-dependent amidation of glutamate to glutamine:

$$
\text { Glutamate }+\mathrm{NH}_{4}^{+}+\mathrm{ATP} \rightarrow \text { Glutamine }+\mathrm{ADP}+\mathrm{P} i
$$

This specific reaction proceeds via a $\gamma$-glutamyl phosphate intermediate, and is dependent upon the presence of divalent cations such as manganese $\left(\mathrm{Mn}^{2+}\right)$.

\section{GLUTAMINE SYNTHETASE IN CHRONIC LIVER FAILURE}

Portal hypertension is a serious complication of chronic liver failure and is relieved either naturally from spontaneous collaterals between the portal vein and the systemic venous circulation, or surgically (as treatment) with a portacaval anastomosis or more commonly used today, transjugular intrahepatic portosystemic shunt (TIPS). In either case, portal-systemic shunting results in an increase in blood and brain ammonia, and hepatic encephalopathy developes.

In rats, 4 weeks following portacaval anastomosis, Girard et al. (1993) found an increase in brain ammonia and a concomitant decrease of GS activity in the brain. This finding was region selective, being observed in 3/7 brain regions (cerebral cortex, cerebellum, and hippocampus) (Fig. 1) with no change found in the other regions. Using the same model, studies by Butterworth et al. (1988) revealed a similar reduction in GS activity in cerebral cortex, and more recently Desjardins et al. (1999) reported similar findings in both cerebral cortex and cerebellum. Cooper et al. (1985) on the other hand did not find a decrease in GS activity and found no change in whole brain from portacaval-shunted rats. Others were unable to detect any significant increase in net glutamine synthesis in brain, following portacaval anastomosis (Cremer et al., 1975; Ukida et al., 1988). Many possible explanations have been forwarded (see later) to explain why the capacity of brain GS to remove ammonia is not increased to compensate for the elevated brain ammonia concentrations, in chronic liver failure. Cooper et al. (1985) showed that GS activity in the brain operates at near maximal capacity under normal physiological conditions and that induction does not occur. This lack of induction or, even worse, decrease of brain GS activity is a likely explanation for the precipitously high levels of brain ammonia in conditions of chronic liver failure (Butterworth et al., 1988). 
Rose, C. \& Felipo, V., 2005. Limited capacity for ammonia removal by brain in chronic liver failure: potential role of nitric oxide. Metabolic brain disease, 20(4), p.275-83.

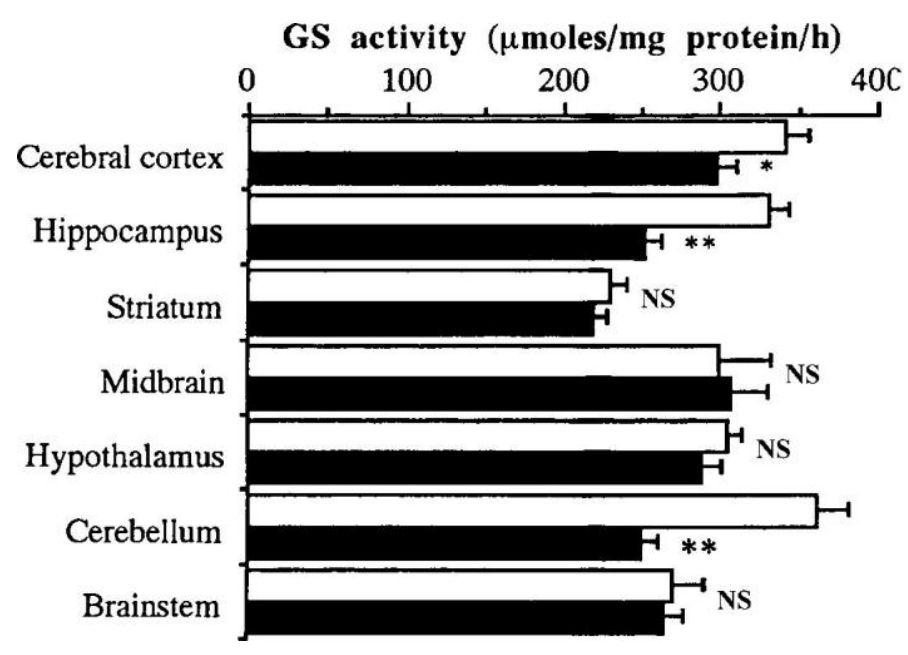

Figure 1. Effect of portacaval anastomosis on glutamine synthetase (GS) activity in different brain regions. White bars, sham-operated; black bars, portacaval anastomosis. The asterisk $\left({ }^{*}\right)$ indicates significantly different from sham-operated; NS, nonsignificant (modified from Girard et al., 1993).

FACTORS INVOLVED IN LIMITING GLUTAMINE SYNTHETASE CAPACITY TO REMOVE AMMONIA

\section{Cell (Astrocyte) Loss}

Chronic liver failure does not result in significant loss of neural cells (i.e., neurons or astrocytes) in the brain (Butterworth, 2003). It is therefore highly unlikely that the reductions in the activity of GS in brain reported following portacaval anastomosis are the consequence of the loss of cells rich in this enzyme, since neuropathologic studies do not reveal evidence of astrocytic cell loss. However, alterations of the functional integrity of astrocytes have been described (Norenberg, 1987).

\section{Decreased Protein or Gene Expression}

It has been shown that the loss of activity of the GS enzyme is not associated with the loss of GS protein or gene expression in the brains of portacaval-shunted rats (Desjardins et al., 1999).

\section{Limited Concentrations of Glutamine Synthetase Enzyme Substrate}

With increasing concentrations of ammonia in the brain during chronic liver failure, it is obvious that the amidation of glutamate to glutamine is not limited by the substrate ammonia in the pathogenesis of hepatic encephalopathy. However, many other factors may be altered, resulting in a decrease or non-induction of GS in astrocytes.

Glutamate is the substrate for GS, and therefore a decrease or increase in intracellular glutamate may affect GS activity. Termination of the action of glutamate in the synaptic cleft relies to a significant degree on its reuptake by high-affinity glutamate transporters 
Rose, C. \& Felipo, V., 2005. Limited capacity for ammonia removal by brain in chronic liver failure: potential role of nitric oxide. Metabolic brain disease, 20(4), p.275-83.

into perineuronal astrocytes where glutamate is transformed via GS into glutamine. Studies in astrocytes cultured from different brain regions of the rat demonstrate that the capacity for glutamate uptake correlates with regional GS activities (Hansson et al., 1986). In chronic liver failure, using in vivo microdialysis, no increase in extracellular glutamate was observed in cerebral cortex (Rao et al., 1995) or striatum (Tossman et al., 1983) of rats with portacaval anastomosis. It has been demonstrated that chronic hyperammonemia results in a region-selective loss of glutamate transporter sites. However, Desjardins and Butterworth found no change in gene expression of the astrocytic glutamate transporters EAAC-1 and EAAC-2 in cerebral cortex of rats with portacaval anastomosis (unpublished results). On the contrary, Suarez et al. (2000) described a significant loss of both astrocytic and neuronal glutamate transporter expression in cerebellum of portacaval-shunted rats (Suarez et al., 2000). In brain tissue, concentrations of glutamate have been found to be decreased in portacaval-shunted rats (Giguere and Butterworth, 1984; Hindfelt et al., 1977; Gjedde et al., 1978) (Fig. 2). Together this data provides evidence to support that glutamate availability may be limited in some brain structures of portacaval-shunted rats. On the countray, whether concentrations of glutamate in the astrocyte compartment (available to GS) are reduced remains unclear.

\begin{tabular}{|c|c|c|c|}
\hline \multirow[b]{3}{*}{ Brain Region } & \multicolumn{3}{|c|}{ Glutamate Concentration } \\
\hline & \multirow[b]{2}{*}{$\begin{array}{c}\text { Sham Operated } \\
\mu \mathrm{mol} / \mathrm{g}\end{array}$} & \multicolumn{2}{|c|}{ Portacaval Shunted } \\
\hline & & $\mu \mathrm{mol} / \mathrm{g}$ & $\%$ Sham Operated \\
\hline Cerebral cortex & $11.35 \pm 1.46$ & $5.67 \pm 1.69^{*}$ & $49.96^{*}$ \\
\hline Hippocampus & $8.55 \pm 1.32$ & $9.27 \pm 1.52$ & 108.42 \\
\hline Striatum & $7.96 \pm 2.90$ & $14.54 \pm 2.12 *$ & $182.66^{*}$ \\
\hline Midbrain & $6.89 \pm 1.15$ & $8.60 \pm 0.68$ & 124.82 \\
\hline Hypothalamus & $7.94 \pm 0.71$ & $4.68 \pm 1.20^{*}$ & $58.94 *$ \\
\hline Cerebellum & $8.75 \pm 1.43$ & $13.91 \pm 1.67^{*}$ & $158.97^{*}$ \\
\hline Medulla-pons & $6.48 \pm 0.94$ & $4.60 \pm 0.80^{*}$ & $70.99 *$ \\
\hline
\end{tabular}

Figure 2. Brain glutamate concentrations following portacaval anastomosis. Results are expressed in $\mu \mathrm{mol} / \mathrm{g}$ wet weight tissue as mean $\pm \mathrm{SD}$. The asterisk $\left({ }^{*}\right)$ indicates significantly different from sham-operated (modified from Giguere and Butterworth, 1984).

Each of the 12 active sites of GS requires two $\mathrm{Mn}^{+}$ions, a metallic ion whose avail-ability is not limited in chronic liver failure. On the contrary, it has been demonstrated that increased manganese deposition occurs in the brain (more specifically in the basal ganglia) of patients with chonic liver failure (Rose et al., 1999) and with spontaneous or surgically-induced portal-systemic shunting (Spahr et al., 1996). Similar observations were found in rats with both portal-systemic shunting and liver dysfunction (Fig. 3). These results suggest that in chronic liver failure, manganese availability is adequate to maintain GS activity.

GS is an ATP-dependent enzyme. Although ammonia has been shown to inhibit $\alpha$ ketoglutarate dehydrogenase in the tricarboxylic acid (TCA) cycle with the potential to 
Rose, C. \& Felipo, V., 2005. Limited capacity for ammonia removal by brain in chronic liver failure: potential role of nitric oxide. Metabolic brain disease, 20(4), p.275-83.

cause energy impairment, no evidence of energy failure has been reported in the brains of rats with chronic liver failure (Hindfelt et al., 1977).

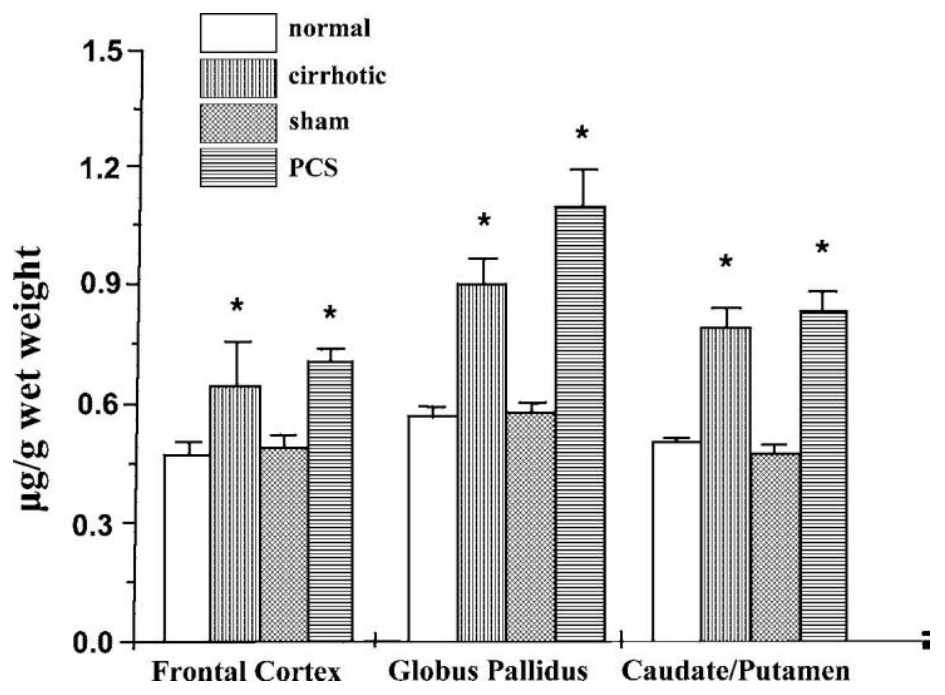

Figure 3. Manganese concentration in rat brain tissue. Data are expressed as mean \pm SEM. The asterisk $\left({ }^{*}\right)$ indicates significantly different from normal and sham-operated (modified from Rose et al., 1999).

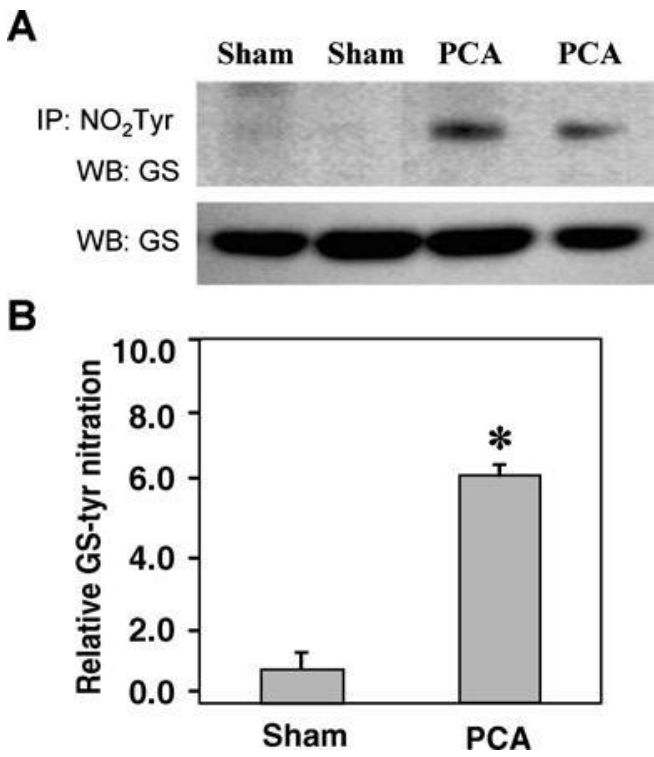

Figure 4. GS nitration in brain in chronic liver failure. PCA rats were sacrificed 4 weeks following portacaval anastomosis. (A) Brain proteins were precipitated with the anti-3 -nitrotyrosine antibody and analyzed using Western blot for the presence of tyrosine-nitrated GS ( $\mathrm{NO}_{2}$ Tyr-GS). (B) Densimetric quantification of tyrosine nitration of GS (modified from Schliess et al., 2002).

There is recent evidence to suggest that a decrease in GS activity can also result from nitration of its tyrosine residues, leading to a loss of enzymatic activity. Ammonia- 
Rose, C. \& Felipo, V., 2005. Limited capacity for ammonia removal by brain in chronic liver failure: potential role of nitric oxide. Metabolic brain disease, 20(4), p.275-83.

induced tyrosine nitration of many proteins has been demonstrated in both in vivo (4week portacaval-shunted rats) (Fig. 4) and in vitro models of hyperammonemia (Schliess et al., 2002). It has been demonstrated in vitro (cultured astrocytes) that an increase in GS tyrosine nitration induced by ammonia is accompanied with a $\sim 30 \%$ decrease in GS activity (Schliess et al., 2002). Tyrosine nitration is mediated by reactive nitrogen species such as peroxynitrite anion $\left(\mathrm{ONOO}^{-}\right)$and nitrogen dioxide $\left({ }^{\bullet} \mathrm{NO} 2\right)$, formed as secondary products of nitric oxide $(\bullet$ NO) metabolism in the presence of oxidants includ-ing superoxide radicals $\left(\mathrm{O}_{2}{ }^{-}\right)$, and hydrogen peroxide $\left(\mathrm{H}_{2} \mathrm{O}_{2}\right)$. ${ }^{\bullet} \mathrm{NO}$ diffuses freely across cell membranes and is believed to play a major role in the nitration of GS. Nitric ox-ide synthase (NOS) catalyses the production of ${ }^{\bullet}$ NO with the oxidation of L-arginine to L-citrulline.

Arginine $+2 \mathrm{NADPH}+2 \mathrm{O}_{2} \rightarrow$ Citrulline $+\mathrm{NADP}+2 \mathrm{H}_{2} \mathrm{O}$

An increase in ${ }^{\bullet} \mathrm{NO}$ production could lead to increased production of reactive nitrogen species and hence nitration of GS, lowering its capacity to remove ammonia.

L-Arginine is the obligate substrate for NOS reaction; its intracellular availability depends exclusively on its transport. There is convincing evidence for a stimulatory effect of ammonia on cellular L-arginine uptake. For example, hyperammonemia increases the transport of $\mathrm{L}-\left[{ }^{3} \mathrm{H}\right]$ arginine into synaptosomes of portacaval-shunted rats, and precipitation of severe encephalopathy following ammonia administration to these rats results in a further increase in synaptosomal $1-\left[{ }^{3} \mathrm{H}\right]$ arginine uptake (Rao et al., 1997a). Furthermore, hyperammonemia without liver dysfunction also stimulates arginine uptake by increasing the $V_{\max }$ without changing the affinity of L-arginine for the transporter. It was demonstrated that increased NOS activity was not responsible for the increased L-arginine uptake (Rao et al., 1997a). None of the NOS inhibitors studied (LNAME, L-NOARG, L-NMMA) had any effect on the ammonia-induced increase in synaptosomal L-arginine uptake, suggesting that ammonia directly activates the transport of l-arginine and that inhibition of NOS has little, if any, effect on this process. Furthermore, Rao et al. (1995) found that increased NOS activities were the consequence of increased availability of the enzyme substrate L-arginine. Overall, ammonia-induced L-arginine uptake is an important pathway in regulating NOS activity.

There are three different genes encoding NOS: (1) nNOS (NOS-1), which until recently, was considered to be expressed only in neurons; (2) iNOS (NOS-2), the inducible form, usually found in macrophages and turned on by an appropriate stimulus; and (3) eNOS $(N O S-3)$ found in the endothelial cells that line the lumen of blood vessels. $n N O S$ and eNOS are constitutively expressed and synthesize ${ }^{\bullet} \mathrm{NO}$ in response to increases in intracellular calcium. Rao et al. (1997b) reported an increased nNOS protein and mRNA expression in the brains of portacaval-shunted rats (Fig. 5). 
Calcium is an important regulator of NOS, acting through the binding of calmodulin. Felipo and colleagues demonstrated that ammonia-induced increases of NOS activity are dependent upon $\mathrm{N}$-methyl $\mathrm{D}$-aspartate (NMDA) receptor activation, resulting in an opening of the ion channel and entry of calcium. This leads to activation of soluble guanylate cyclase, leading to an increased formation of cyclic guanosine monophosphate (cGMP). Part of the cGMP formed is released into the extracellular space where it can be collected and served as a marker of NMDA-receptor-mediated NOS activity. This pathway is blocked by the pre-treatment of MK-801, a glutamate (NMDA) receptor antagonist (Hermenegildo et al., 1996, 2000). Overactivation of NMDA receptors leading to an increase in NMDA/NOS/cGMP pathway has been shown to occur in acute hyperammonemia (Marcaida et al., 1992; Montoliu et al., 2002). However, in chronic liver failure, overactivation of NMDA recep-tors does not occur. Peterson et al. demonstrated a region-selective reduction of NMDA receptor densities in brains of portacaval-shunted rats (Peterson et al., 1990). It is therefore unlikely that NMDAreceptor-mediated increases of the NO-cGMP signal transduction pathway are responsible for GS nitration in chronic liver failure.

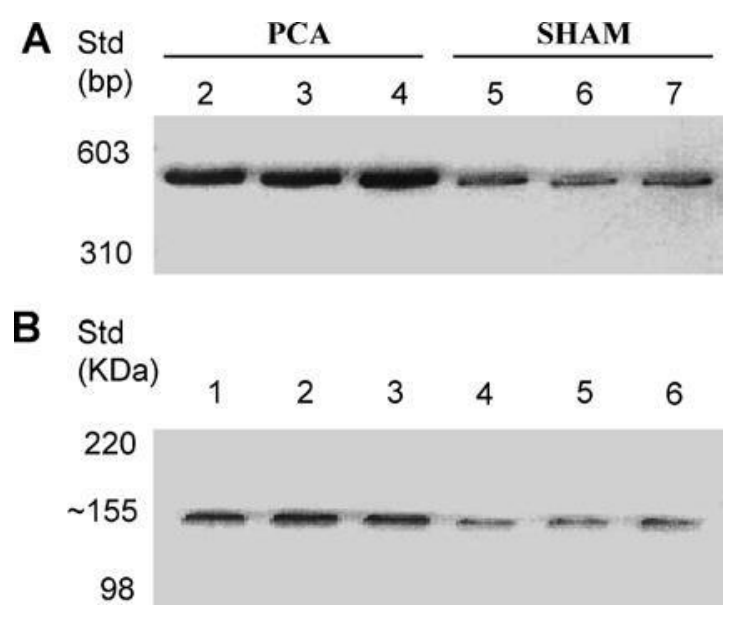

Figure 5. (A) nNOS expression (total RNA) in cerebellum of 4-week portacaval-shunted (lane 2-4) and sham-operated (lanes 5-7) rats. (B) Western blot analysis of cytosol protein from cerebellum of 4-week portacaval-shunted (lanes1-3) and sham-operated (lanes 4-6) rats (modified from Rao et al., 1997b). 


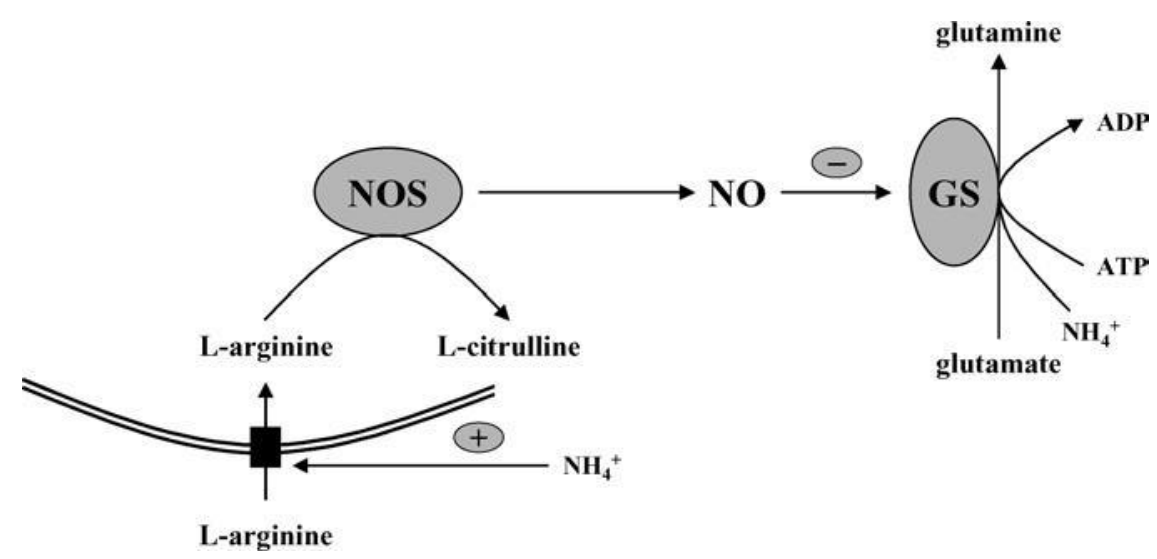

Figure 6. Hypothesis whereby ammonia stimulates L-arginine uptake into the cell, which activates NOS, resulting in an increased production of NO and consequently tyrosine nitration of GS. Even though GS is localized in astrocytes, the source of NO is yet to be defined, as NOS is found in both neurons and astrocytes (Baltrons and Garcia, 2001).

In summary, under hyperammonemic conditions, the brain is not capable of increas-ing its capacity to remove ammonia via its principal ammonia-removing mechanism, the astrocytic GS pathway. A review of currently available evidence supports the view that the reduction in GS capacity in brain (or lack of its induction, depending upon the brain region) in chronic liver failure does not result from a limitation in availability of substrates $\left(\mathrm{NH}_{4}{ }^{+}\right.$, glutamate) or cofactors (ATP, $\left.\mathrm{Mn}^{2+}\right)$. The most likely explanation is GS nitration resulting from ammonia-induced increased synthesis of NO. This later mechanism appears to be independent of NMDA activation but dependent upon Larginine (Fig. 6).

\section{ACKNOWLEDGMENTS}

The authors thank the Canadian Insitutes of Health Research (CIHR) for a postdoctoral fellowship award (to CR).

\section{REFERENCES}

Baltrons, M.A., and Garcia, A. (2001). The nitric oxide/cyclic GMP system in astroglial cells. Prog. Brain Res. 132:325-337.

Butterworth, R.F. (2003). Hepatic encephalopathy. Alcohol Res. Health 27:240-246.

Butterworth, R.F., Girard, G., and Giguere, J.F. (1988). Regional differences in the capacity for ammonia removal by brain following portacaval anastomosis. J. Neurochem. 51:486-490.

Cooper, A.J.L., Mora, S.N., Cruz, N.F., and Gelbard, A.S. (1985). Cerebral ammonia metabolism in hyperam-monemic rats. J. Neurochem. 4:1716-1723. 
Rose, C. \& Felipo, V., 2005. Limited capacity for ammonia removal by brain in chronic liver failure: potential role of nitric oxide. Metabolic brain disease, 20(4), p.275-83.

Cremer J.E., Heath, D.F., Teal, H.M., Woods, M.S., and Cavanagh, J.B. (1975). Some dynamic aspects of brain metabolism in rats given a portacaval anastomosis. Neuropathol. Appl. Neurobiol. 3:293311.

Desjardins, P., Rama Rao, K.V., Michalak, A., Rose, C., and Butterworth, R.F. (1999). Effect of portacaval anastomosis on glutamine synthetase protein and gene expression in brain, liver and skeletal muscle. Metab. Brain Dis. 14:273-280.

Giguere, J.-F., and Butterworth, R.F. (1984). Amino acid changes in regions of the CNS in relation to function in experimental portal-systemic encephalopathy. Neurochem. Res. 9:1309-1321.

Girard, G., Giguere, J.-F., and Butterworth, R.F. (1993). Region-selective reductions in activities of glutamine synthetase in rat brain following portacaval anastomosis. Metab. Brain Dis. 8: 207-215.

Gjedde, A., Lockwood, A.H., Duffy, T.E., and Plum, F. (1978). Cerebral blood flow and metabolism in chronically hyperammonemic rats: Effect of an acute ammonia challenge. Ann. Neurol. 3:325-330.

Hansson, E. (1986). Primary cultures from defined brain areas: III. Effects of seeding time on 3[H]L-glutamate transport and glutamine synthetase activity. Brain Res. 389:203-209.

Hermenegildo, C., Marcaida, G., Montoliu, C., Grisolia, S., Minana, M.D., and Felipo, V. (1996). NMDA receptor antagonists prevent acute ammonia toxicity in mice. Neurochem. Res. 21:1237-1244.

Hermenegildo, C., Monfort, P., and Felipo, V. (2000). Activation of NMDA receptors in rat brain in vivo following acute ammonia intoxication. Characterization by in vivo microdialysis. Hepatology 31:709- 715 .

Hindfelt, B., Plum, F., and Duffy, T.E. (1977). Effect of acute ammonia intoxication on cerebral metabolism in rats with portacaval shunts. J. Clin. Invest. 59:386-396.

Marcaida, G., Felipo, V., Hermenegildo, C., Minana, M.D., and Grisolia, S. (1992). Acute ammonia toxicity is mediated by the NMDA type of glutamate receptors. FEBS Lett. 296:67-68.

Montoliu, C., Kosenko, E., Erceg, S., Canales, J.J., and Felipo, V. (2002). Molecular mechanism of acute ammonia toxicity: Role of NMDA receptors. Neurochem. Int. 41:95-102.

Norenberg, M.D. (1987). The role of astrocytes in hepatic encephalopathy. Neurochem. Pathol. 6:13-33. Peterson, C., Giguere, J.-F., Cotman, C.W., and Butterworth, R.F. (1990). Selective loss of Nmethyl-d-aspartate-sensitive L-[3H]glutamate binding sites in rat brain following portacaval anastomosis. J. Neurochem. 55:386- 390.

Rao, V.L.R., Audet, R.M., Butterworth, R.F. (1995). Selective alterations of extracellular brain amino acids in relation to function in experimental portal-systemic encephalopathy: Results of an in vivo microdialysis study. J. Neurochem. 65:1221-1228.

Rao, V.L.R., Audet, R.M., and Butterworth, R.F. (1997a). Portacaval shunting and hyperammonemia stimulate the uptake of $1-[3 \mathrm{H}]$ arginine but not the $\mathrm{L}-[3 \mathrm{H}]$ nitroarginine into rat brain synaptosomes. J. Neurochem. 68:337-343.

Rao, V.L.R., Audet, R.M., and Butterworth, R.F. (1997b). Increased neuronal nitric oxide synthase expression in brain following portacaval anastomosis. Brain Res. 765:169-172. 
Rose, C. \& Felipo, V., 2005. Limited capacity for ammonia removal by brain in chronic liver failure: potential role of nitric oxide. Metabolic brain disease, 20(4), p.275-83.

Rose, C., Butterworth, R.F., Zayed, J., Normandin, L., Todd, K., Michalak, A., Spahr, L., Huet, P.-M., and Pomier-Layrargues, G. (1999). Manganese deposition in basal ganglia structures results from both portal-systemic shunting and liver dysfunction. Gastroenterology 117:640-644.

Schliess, F., Gorg, B., Fisher, R., Desjardins, P., Bidmon, H.J., Herrmann, A., Butterworth, R.F., Zilles, K., and Haussinger, D. (2002). Ammonia induces MK-801-sensitive nitration and phosphorylation of protein tyrosine residues in rat astrocytes. FASEB J. 16:739-741.

Suarez, I., Bodega, G., and Fernandez, B. (2000). Modulation of glutamate transporters (GLAST, GLT-1 and EAAC-1) in the rat cerebellum following portacaval anastomosis. Brain Res. 859:293302.

Spahr, L., Butterworth, R.F., Fontaine, S., Bui, L., Therrien, G., Milette, P., Lebrun, L.H., Zayed, J., Leblanc, A., and Pomier-Layrargues, G. (1996). Increased blood manganese in cirrhotic patients: Relationship to pallidal magnetic resonance signal hypertensity and neurological symptoms. Hepatology 24:1116- 1120.

Tossman, U., Delin, A., Eriksson, S., and Ungerstedt, U. (1987). Brain cortical amino acids measured by intrac-erebral dialysis in portacaval shunted rats. Neurochem. Res. 12:265-269.

Tossman, U., Eriksson, S., Delin, A., Hagenfeldt, L., Law, D., Ungerstedt, U. (1983). Brain amino acids measured by intracerebral dialysis in portacaval shunted rats. J. Neurochem. 41:1046-1051.

Ukida, M., Morishita, H., Morimoto, Y., Usui, H., and Nagashima, H. (1988). Limited glutamine synthesis in brains of dogs with a portacaval anastomosis after $15 \mathrm{~N}$-ammonium chloride loading. In (P.B. Soeters, J.H.P. Wilson, A.J. Meijer, E. Holm, eds.), Advances in Ammonia Metabolism and Hepatic Encephalopathy, Excerpta Medica, Amsterdam, pp. 433-438. 